\title{
Androgen receptor expression predicts breast cancer survival: the role of genetic and epigenetic events
}

\author{
Kate M Peters ${ }^{1}$, Stacey L Edwards ${ }^{1}$, Shalima S Nair ${ }^{2}$, Juliet D French ${ }^{1}$, Peter J Bailey ${ }^{1}$, Kathryn Salkield ${ }^{1}$, \\ Sandra Stein ${ }^{3}$, Sarah Wagner ${ }^{3}$, Glenn D Francis ${ }^{3}$, Susan J Clark² and Melissa A Brown ${ }^{1 *}$
}

\begin{abstract}
Background: Breast cancer outcome, including response to therapy, risk of metastasis and survival, is difficult to predict using currently available methods, highlighting the urgent need for more informative biomarkers. Androgen receptor (AR) has been implicated in breast carcinogenesis however its potential to be an informative biomarker has yet to be fully explored. In this study, AR protein levels were determined in a cohort of 73 Grade III invasive breast ductal adenocarcinomas.
\end{abstract}

Methods: The levels of Androgen receptor protein in a cohort of breast tumour samples was determined by immunohistochemistry and the results were compared with clinical characteristics, including survival. The role of defects in the regulation of Androgen receptor gene expression were examined by mutation and methylation screening of the $5^{\prime}$ end of the gene, reporter assays of the $5^{\prime}$ and $3^{\prime}$ end of the AR gene, and searching for miRNAs that may regulate AR gene expression.

Results: AR was expressed in 56\% of tumours and expression was significantly inversely associated with 10-year survival $(P=0.004)$. An investigation into the mechanisms responsible for the loss of AR expression revealed that hypermethylation of the $A R$ promoter is associated with loss of AR expression in breast cancer cells but not in primary breast tumours. In AR negative breast tumours, mutation screening identified the same mutation (T105A) in the $5^{\prime} U T R$ of two AR negative breast cancer patients but not reported in the normal human population. Reporter assay analysis of this mutation however found no evidence for a negative impact on AR $5^{\prime} U T R$ activity. The role of miR-124 in regulating AR expression was also investigated, however no evidence for this was found.

Conclusion: This study highlights the potential for AR expression to be an informative biomarker for breast cancer survival and sets the scene for a more comprehensive investigation of the molecular basis of this phenomenon.

Keywords: Androgen receptor, Prognostic biomarker, Breast cancer, Gene regulation, Promoter methylation, Regulatory mutation, MiRNA

\section{Background}

Breast cancer is a heterogeneous disease comprising tumour subtypes associated with variable clinical characteristics [1]. Variables including tumour size, histological subtype and grade, lymph node status and the expression of estrogen receptor alpha $(E R \alpha)$, progesterone receptor (PR) and human epidermal growth factor

\footnotetext{
* Correspondence: melissa.brown@uq.edu.au

'School of Chemistry and Molecular Biosciences, The University of

Queensland, St. Lucia 4072 Queensland, Australia

Full list of author information is available at the end of the article
}

receptor 2 (HER2) currently assist routine clinical management [2]. However, these factors are limited in their ability to predict individual survival and response to therapy [2]. This is particularly apparent for patients with advanced breast cancer, which is characterised by high histological grade and the presence of lymph node metastases, and has an aggressive clinical course and generally a poor prognosis [2]. Identifying new prognostic biomarkers and the molecular mechanisms underlying breast cancer progression are paramount for 
improving the clinical management of these patients and developing improved therapeutic strategies.

Androgen receptor (AR) is a member of the nuclear receptor superfamily and is known to be involved in a complex network of signalling pathways that collectively regulate cell proliferation [3,4]. Expressed in the normal human mammary gland, where it predominantly localises to the inner layer of epithelial cells lining acini and intralobular ducts [5], the role of AR in normal mammary epithelial biology is unknown. AR has been implicated in breast tumourigenesis, however delineating its precise function has proven difficult with AR-mediated androgenic effects shown to both stimulate and inhibit growth of breast cancer cells [6,7]. The significance of $A R$ in human breast cancer is further emphasized by the recent finding that it can be targeted in estrogen receptor negative breast tumours [8]. Loss of AR expression is associated with early onset, high nuclear grade and negative ER, PR and HER2 expression status in breast tumours $[9,10]$. However, the mechanisms responsible for this loss of AR expression in breast carcinogenesis remain unclear.

The $A R$ gene comprises 9 exons spanning 180.25 kilobases located on chromosome Xq12. Functional analyses have identified two independently regulated transcription initiation sites (TIS), AR-TIS I $(-12 /-11 /-10)$ and $A R$-TIS II $(-1 /+1)$ (Figure 1) [11]. Transcriptional initiation from $A R$-TIS I is dependent on sequences located between positions -17 and +45 and initiation from $A R$ TIS II facilitated by a palindromic homopurine repeat and SP1 binding to a GC-box [12,13]. Additional putative cis-acting elements include HL (helix-loop-helixlike) motifs 1 and 2 [14] and a cAMP responsive element [15]. Two CpG islands (CGI) are also located in

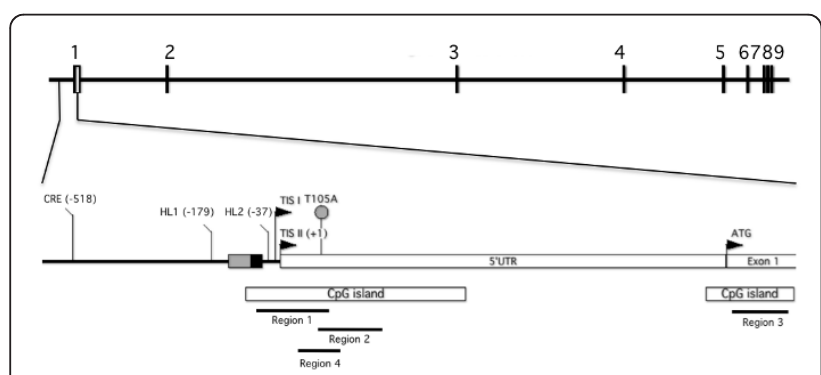

Figure 1 Schematic diagram of the human $A R$ gene. The relative positions of the two transcription initiation sites (TIS I and II) and functionally known motifs; $\mathrm{CpG}$ islands, CAMP responsive element (CRE), helix-loop-helix-like (HL) motifs, a palindromic homopurine repeat (grey box) and GC-box (black box) which contains an SP1 binding site, are indicated (GenBank Accession No. NG009014.1). A grey circle denotes the $T 105 \mathrm{~A}$ alteration identified in two primary breast tumours in the present study. DNA methylation was assessed in Regions 1-4, as denoted by black lines. UTR, untranslated region. ATG, translation start site. the $A R$ promoter and extend into Exon 1. Hypermethylation of these CGI have been shown to silence $A R$ transcription in prostate cancer cells and primary tumours [16]. Genetic alterations in the promoter and 5'untranslated regions (UTR) of the $A R$ gene have been also observed in prostate cancer cell lines, xenografts [17] and in two prostate cancer patients $[18,19]$. In breast cancer, the role of regulatory defects in the AR gene are yet to be fully elucidated.

In this study, we show that loss of AR expression is significantly associated with poor 10 year survival outcome in Grade III invasive breast ductal adenocarcinomas. We then evaluated potential regulatory mechanisms that may account for the loss of AR expression. For the first time we show that DNA hypermethylation in the $A R$ promoter is associated with loss of $A R$ expression in breast cancer cells, although this is not the case in our cohort of tumours from patients with Stage III breast cancer. We subsequently assessed whether somatic mutations in AR regulatory regions or miRNAs bioinformatically predicted to target the human $A R$ 3'UTR might contribute to the observed changes in AR expression.

\section{Results and Discussion}

\section{Low AR protein levels are associated with poor 10-year} survival in patients with Stage III breast cancer

To assess the prognostic value of AR expression in breast cancer patients, IHC analysis was performed in a cohort of 73 Grade III lymph node positive ductal adenocarcinomas from patients with Stage III disease. Patient and tumour characteristics are summarised in Table 1. The patients ranged in age from 30 to 94 years (mean, 54 years); with the majority of patients (97\%) aged over 35 years. AR expression was detected in 56\% $(n=41)$ of primary breast tumours. Positive expression of ER, PR and HER2 was also observed in 55.5\% ( $\mathrm{n}=$ $40), 40 \%(n=29)$ and $21.7 \%(n=15)$ of breast tumours, respectively. In AR-negative tumours, the majority (72\%, $\mathrm{n}=23 ; 87 \%, \mathrm{n}=27 ; 86.6 \%, \mathrm{n}=26$ ) were also $\mathrm{ER}, \mathrm{PR}$ and HER2 negative, respectively. The authors acknowledge the potential limitations of TMA analysis, given the inherent heterogeneity of tumour samples, but note the evidence that there is a high concordance between TMA cores and whole sections [20]. In addition the impact was further minimized by analysing at least two cores from each tumour, in accordance with the correlation nomograms developed by Karlsson et al., 2009 [21]. AR expression was a significant prognostic factor for overall patient survival $(\mathrm{P}=0.004)$ (Figure 2$)$. The 10 year survival of patients with AR positive tumours was $52 \%$ versus $22 \%$ for patients with AR negative tumours. This finding is consistent with previous studies in a diversity of breast cancer patient populations wherein a 
Table 1 Patient and tumour characteristics*

\begin{tabular}{lcc}
\hline $\begin{array}{l}\text { Factor } \\
\text { Age (years) }\end{array}$ & $\begin{array}{c}\text { AR negative } \mathbf{n}=\mathbf{3 2} \\
\text { (\%) }\end{array}$ & $\begin{array}{c}\text { AR positiven }=\mathbf{4 1} \\
\text { (\%) }\end{array}$ \\
\hline$\leq 35$ & $1(3.0)$ & $1(2.4)$ \\
\hline$\geq 35$ & $31(97.0)$ & $40(97.6)$ \\
\hline Estrogen receptor & $23(72.0)$ & $9(22.5)$ \\
\hline Negative & $9(28.0)$ & $31(77.5)$ \\
\hline Positive & & $16(39.0)$ \\
\hline Progesterone & $27(87.0)$ & $25(61.0)$ \\
\hline receptor & $4(13.0)$ & $25(75.8)$ \\
\hline Negative & & $8(24.2)$ \\
\hline Positive & $18(85.7)$ & $30(90.9)$ \\
\hline HeR2 & $3(14.3)$ & $3(9.1)$ \\
\hline Negative & & \\
\hline Positive & $6(28.6)$ & \\
\hline Triple negative ${ }^{a}$ & $15(71.4)$ & No
\end{tabular}

*All patients were diagnosed with Stage III disease, as defined in the Materials and Methods section of the manuscript

${ }^{a}$ Triple negative breast cancer represents tumours displaying negative expression for estrogen receptor, progesterone receptor and HER2 by IHC. AR, androgen receptor; HER2, human epidermal growth factor receptor type 2.

significant association between AR expression and age at diagnosis, nuclear grade, recurrence-free survival was observed [9,10,22-24].

\section{DNA methylation of the $A R$ promoter is associated with} low $A R$ mRNA levels in breast cancer cell lines

To investigate potential mechanisms responsible for the loss of AR expression, we evaluated the methylation status of the $A R$ promoter region and $A R$ expression levels

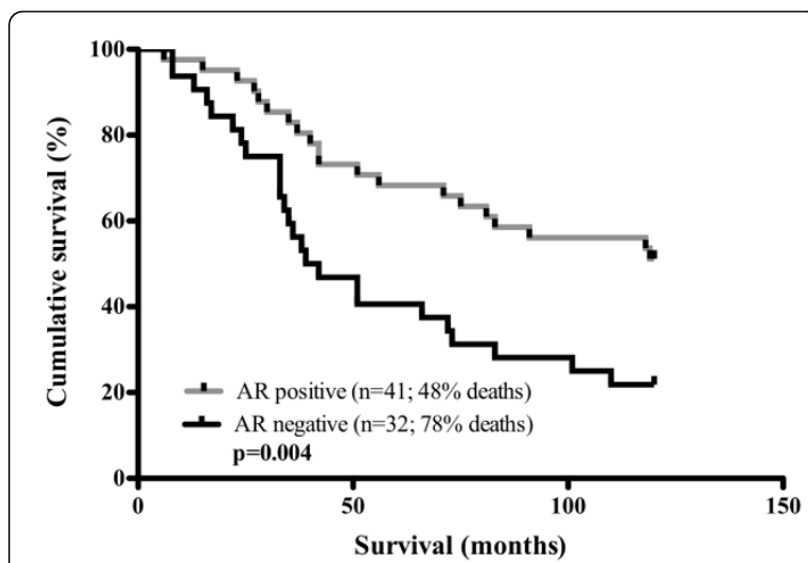

Figure 2 Impact of Androgen Receptor expression on breast cancer survival. Kaplan-Meier estimates of 10 year survival of patients by AR expression for primary tumours. $n$ denotes the number of patient samples. in breast cancer cell lines. DNA methylation was determined by MS-HRM analysis of bisulfite treated DNA in three regions; Regions 1 and 2 correspond to the CpG island in the $A R$ minimal promoter [14] and Region 3 corresponds to the $\mathrm{CpG}$ island at the translational start site (Figure 1). DNA methylation was also assessed in a further region, Region 4, by Sequenom MassARRAY (Figure 1). Cell lines, MDAMB231, MCF7, MDAMB157, MDAMB468 and MDAMB436 all showed between 25$100 \%$ methylation in at least 2 of regions analysed (Figure 3a). Notably, methylation of the $A R$ promoter region was associated with the level of $A R$ mRNA (Figure 3a and $3 \mathrm{~b}$ ). A similar association has been observed in prostate cancer, where treatment of prostate cancer cell lines that display $A R$ hypermethylation with the demethylating agent 5-aza-2'-deoxycytidine induces the re-expression and function of AR [25].

\section{$A R$ promoter methylation is not associated with low $A R$} protein levels in primary breast tumours

To examine whether $A R$ promoter methylation is also associated with loss of AR protein levels in breast tumours, the DNA methylation status of Region 4, which contains six CpG dinucleotides, was assessed by

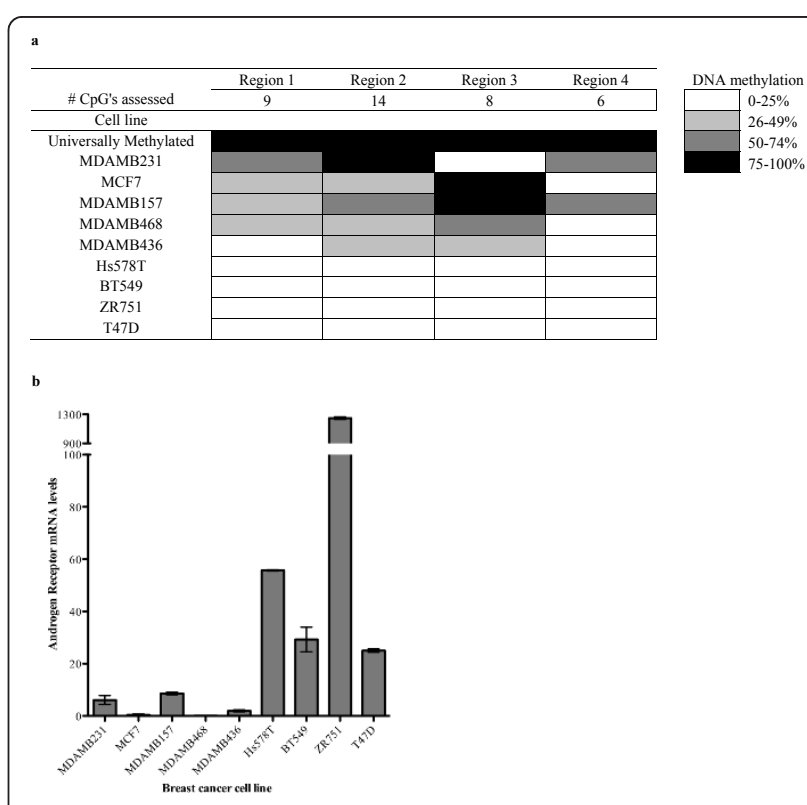

Figure 3 DNA methylation of the $A R$ gene is associated with loss of AR protein expression in breast cancer cells. (a) DNA methylation status of breast cancer cell lines. Three regions of $A R$ were assessed by MS-HRM, overlapping Regions 1 and 2 in the AR minimal promoter and Region 3 at the translational start site (refer to Figure 1). Region 4 was assessed by Sequenom MassARRAY. Data represent the average of two independent experiments. (b) AR mRNA expression in breast cancer cell lines was assessed by qPCR. Expression is shown relative to $\beta$-actin and bars represent the mean \pm standard deviation of two independent experiments. 
Sequenom MassARRAY. Sequenom was chosen for this analysis as it can analyse methylation at each individual CpG dinucleotide, reliably detecting methylation as low as $5 \%$, in a high-throughput manner [26]. Primer design constraints meant that Region 4 of the $A R$ promoter (Figure 1) was selected for analysis, with DNA methylation of overlapping Regions 1 and 2 associated with $A R$ mRNA levels in breast cancer cell lines. DNA methylation was observed in breast cancer patients at each of the six CpGs (Figure 4). However, with the exception of CpG's 1-3, at which methylation in most tumours was greater than $30 \%$, for the most part only low level methylation $(<30 \%)$ was observed. Furthermore, no significant association was observed in the average methylation between AR negative and AR positive primary breast tumours in our cohort at any of the six CpG dinucleotides examined.

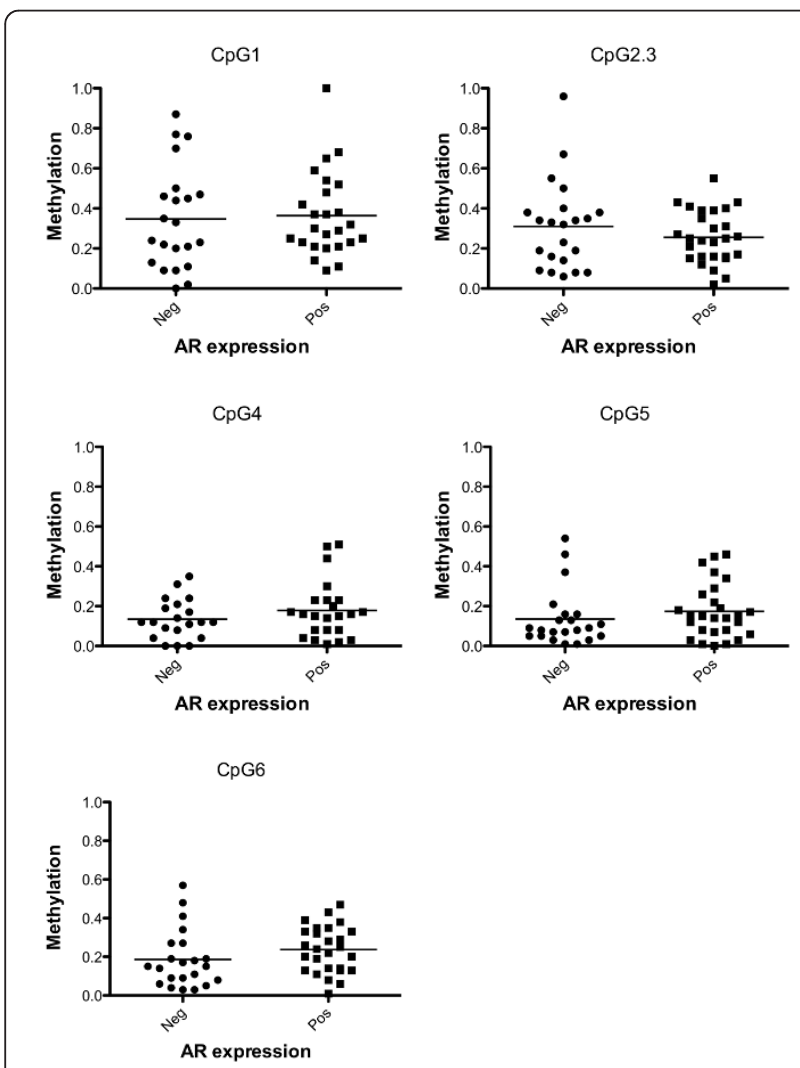

Figure 4 DNA methylation of an $115 \mathrm{bp}$ region in the $A R$ promoter is not associated with loss of AR protein expression in a selective group of primary breast tumours. Methylation of six CPG dinucleotides in the AR promoter of AR positive (Pos) and negative (Neg) Grade III breast tumours as determined by Sequenom MassARRAY (Region 4, Figure 1). Due to cleavage patterns, the average methylation was determined for $\mathrm{CpG}$ dinucleotides 2 and 3. Each dot represents an individual patient. A methylation value of 1.0 indicates a fully methylated amplicon, while a value of 0.0 indicates a fully unmethylated amplicon. Horizontal lines represent average cohort methylation.
There are several plausible explanations for the lack of association between promoter methylation and expression in primary breast tumours. There is the possibility that a region outside that examined shows expression associated methylation and indeed, there was an incomplete association between Region 4 methylation and AR mRNA levels in breast cancer cells. Additionally, another mechanism such as somatic mutation of these regions, or aberrant targeting by a miRNA, may be involved.

\section{Identification of somatic mutations in the $A R 5^{\prime}$ UTR in AR negative breast tumours}

Somatic mutation of regulatory regions of the $A R$ gene is another potential mechanism responsible for reduced AR expression in breast tumours. To address this possibility, we sequenced the 5 ' regulatory region of $A R(-659$ to +280$)$ in breast cancer cell lines. Our results revealed no sequence variations in MDAMB157, MDAMB231, MDAMB436, MDAMB468, MCF7, T47D, ZR75-1, Hs578T and BT549 cells. We also sequenced the $A R$ promoter region from -6 to +133 in 32 primary breast tumours. Amplification of the $A R$ promoter region from -165 to -7 , which corresponds to the homopurine repeat and GC box (Figure 1), revealed a mutation (mRNA pos 105, T > A, Figure 1), mapping to the $A R 5^{\prime} \mathrm{UTR}$, in two patients. This sequence variation does not correspond to any known SNPs (GRCh37 reference primary assembly, http://www.ncbi.nlm.nih.gov/sites/entrez?db=snp) or, to our knowledge, any previously reported $A R$ variants.

To investigate the potential significance of the $A R$ 5'UTR T105A variant, we performed bioinformatics analysis on the wild-type and variant sequence. In the wildtype sequence the $\mathrm{T}$ position is invariant in mammals and is a component of the binding site for RNApolII (based on ChIP-seq data) and the predicted and conserved binding site for several transcription factors, including RUNX1, En1 and Pax6. Based on the currently available ChIP-seq data however, there is currently no evidence that these transcription factors bind to this sequence in vivo. The substitution from $\mathrm{T}$ to $\mathrm{A}$ results in an abolishment of these predicted sites and the creation of predicted and conserved binding sites for NHLH1 (data not shown).

To experimentally address the effect of this variant on 5'UTR activity, we fused the 5'UTR upstream of the firefly cDNA and downstream of either the $A R$ or the SV40 promoter, in pGL3-basic and pGL3-promoter vectors, respectively. The $A R$ 5'UTR T105A sequence variant did not have a negative impact on SV40 or $A R$ driven reporter activity in either MCF7 or T47D cells (Figure 5). Instead, the $A R$ 5'UTR T105A sequence variant actually increased $A R$ reporter driven activity in T47D cells ( $\mathrm{P}=$ 0.0001) (Figure 5). 


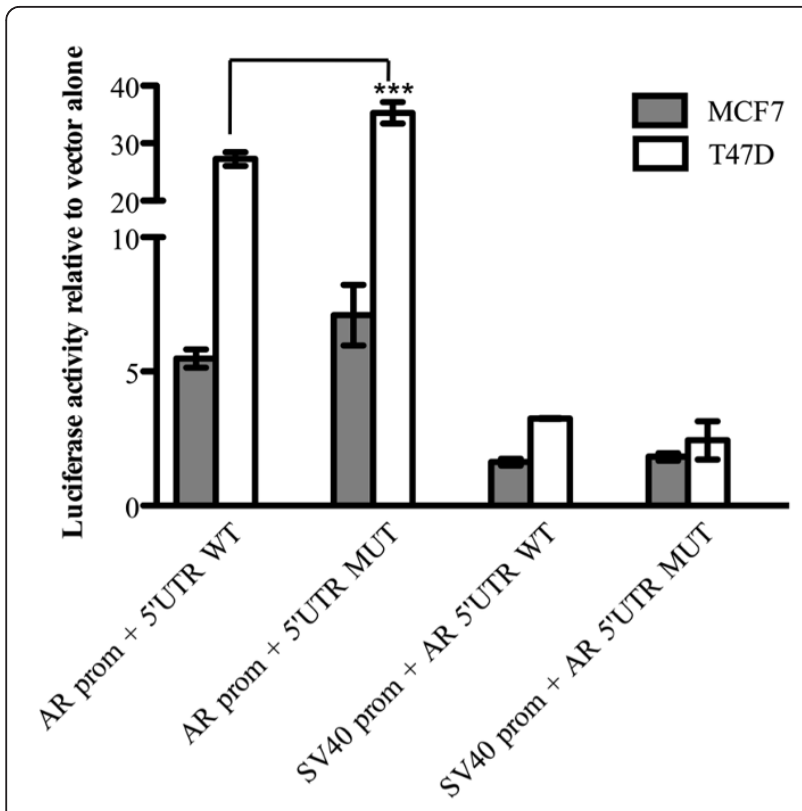

Figure 5 Impact of the $A R$ T105A $5^{\prime}$ UTR variant. Luciferase reporter activities of $\mathrm{pGL} 3$ basic with the $A R$ promoter (AR prom) and PGL3 promoter vector which contains the SV40 promoter (SV40 prom) together with either the wild-type (WT) or T105A mutant (MT) AR 5'UTR sequence in MCF7 and T47D cells. Data is shown relative to the respective empty vector \pm the standard error of the mean (SEM) and was generated from three independent experiments. ${ }^{* *} P=0.0001$

Somatic mutation analysis of AR negative breast tumours identified the same alteration (chrX:66680703, mRNA pos 105, T > A) in the AR 5'UTR of two breast tumours. Examination of the functional importance of this sequence variation however revealed that there was no negative impact on the activity of the $A R 5^{\prime}$ UTR in MCF7 or T47D cells. This suggests that this mutation is unlikely to negatively affect the regulation of AR expression. However, particularly given the AR 5'UTR T105A sequence variant significantly increased $A R$ reporter driven activity in T47D cells, more complex studies, such as analysing the consequence of this mutation in the context of the entire $A R$ gene and AR protein expression, will be required to firmly establish this. Although further analysis of our tumours was constrained by the availability and nature of the FFPE tumour material, it is plausible that mutations that effect AR expression exist outside the promoter region examined, particularly in regions upstream of the $A R 5^{\prime} U T R$ and in the $A R$ 3'UTR, which are reported to contain putative regulatory elements involved in controlling mRNA stability [27]. A recent epidemiological meta-analysis of the $A R$ gene concluded that common polymorphisms in the $A R$ gene are not associated with breast cancer risk among Caucasian women [19]. However, the functional significance of these variants, the AR expression status and survival outcome of these patients was not considered in this study.

\section{miR-124 does not regulate the $A R 3^{\prime} U T R$ in breast cancer} cells

MiRNAs are small non-coding RNAs of $\sim 20 \mathrm{nt}$ in length that are capable of modulating gene expression post-transcriptionally. Many have been shown to act as either oncogenes or tumour suppressor genes that are crucial to the development of breast cancer metastasis and survival outcome [28]. To address the possibility that altered expression of AR is mediated by differential expression of miRNAs, we screened the $A R$ 3'UTR for potential miRNA target sites. Bioinformatic analysis revealed that miR-124 was the only miRNA predicted to target the human AR 3'UTR using miRanda and TargetScan. To examine whether miR-124 regulates the expression of the $A R$ transcript, we used a reporter gene assay. MCF7 and T47D cells were transfected with pcDNA 3.1(+)-mir-124 vector and expression of miR-124 verified. Cells transfected with pcDNA 3.1(+)-mir-124 expressed high levels of mature miR-124 at $12,24,39$ and $48 \mathrm{hr}$ time intervals post-transfection, whereas no endogenous expression was detected in control-transfected cells (Figure 6a). For luciferase assays, we co-transfected MCF7 and T47D cells with pcDNA 3.1(+)-mir-124 vector with the pSG5-AR 3'UTR vector. The introduction of the $A R$ 3'UTR into pSG5 luc significantly increased reporter activity in MCF7 ( $\mathrm{P}=0.007)$ but not in T47D cells (Figure 6b). miR-124 overexpression did not alter luciferase activity of the $A R$ 3'UTR construct in any of the cell lines examined (Figure 6b). These results suggest that miR-124 is unlikely to regulate AR expression in these cells.

MiR-124 was the only miRNA predicted by two commonly used algorithms to target the AR 3'UTR. However, over-expression of miR-124 did not regulate AR expression in MCF7 or T47D cells, which otherwise display no endogenous expression of this miRNA. This suggests that either miR-124 is unable to regulate AR in this particular experimental system, or that the prediction not correct. There is certainly evidence suggesting that although a plethora of targets are predicted for miRNAs, that many of these are false positives [29]. There have been reports of other miRNAs regulating the expression of AR, including miR-488 [30]. It would be of interest to determine whether there is an association between the levels of these miRNAs and AR in Stage III breast cancer, and whether these miRNAs have the potential to be informative biomarkers or therapeutic targets for this disease. Interestingly, the inclusion of the $A R$ 3'UTR significantly increased reporter activity in MCF7 but not in T47D cells suggesting the potential 


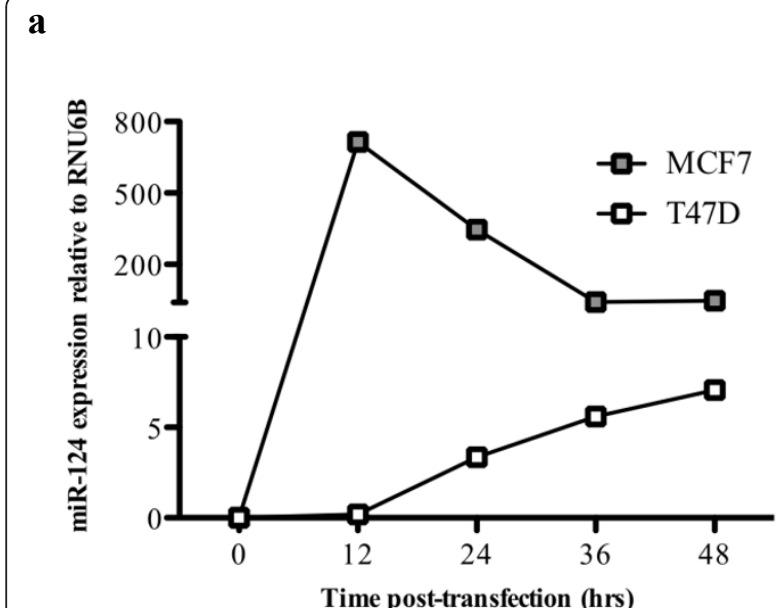

b

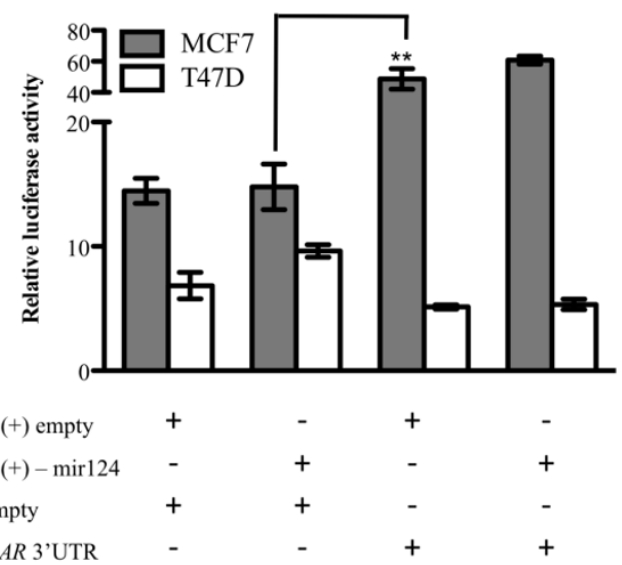

Figure 6 miR-124 does not target the $A R$ 3'UTR in breast cancer cells. (a) miR-124 expression in MCF7 and T47D cells was assessed by qPCR following transfection with pcDNA 3.1(+)-mir-124. Expression is shown relative to RNU6B and dots represent the mean \pm standard deviation of two independent experiments. (b) Luciferase reporter activities relative to the Renilla internal control \pm the standard error of the mean (SEM) are shown. Data were generated from three independent experiments. ${ }^{* *} \mathrm{P}=0.007$.

cell specific importance of this region in the post-transcriptional regulation in breast cancer cells.

\section{Conclusions}

In this paper we show that AR expression is significantly associated with 10-year survival outcome in patients with Stage III breast cancer. To predict and potentially address the poor survival outcome of patients with AR negative breast tumours it is important to understand the mechanism underlying the reduced AR expression.
Here we demonstrate for the first time that hypermethylation of sections of the $A R 5^{\prime}$ regulatory region is associated with loss of AR expression in breast cancer cell lines, although not in a small set primary tumours. We describe a new somatic mutation in the AR 5'UTR which is found in two independent tumours and is not a normal polymorphism. This study highlights the potential for AR expression to be an informative prognostic biomarker for breast cancer survival and sets the scene for a more comprehensive investigation of the molecular basis of this phenomenon.

\section{Methods}

\section{Breast cancer cell lines}

Breast cancer cell lines MDAMB157 (ER-PR-), MDAMB231 (ER-PR-HER2-), MDAMB436 (ER-PR-), MDAMB468 (ER-PR-), MCF7 (ER + PR + HER2-), T47D (ER + PR + HER2-), ZR75-1 (ER + PR-), Hs578T (ER-PR-HER2-), and BT549 (ER-PR-) were obtained from American Type Culture Collection (ATCC) and cultured according to the manufacturer's recommendations. Hormone receptor status sourced from [31].

\section{Clinical samples}

Primary breast tumours were sourced from the Princess Alexandra Hospital (Brisbane, Australia) following procedures endorsed by both The National Ethics Application process of the National Health and Medical Research Council of Australia (http://www.neaf.gov.au) and The University of Queensland Human Ethics committee. Tumour tissues were formalin fixed and paraffin embedded (FFPE), sectioned and stained using hematoxylin and eosin (H\&E), and confirmed by a qualified pathologist (G.D.F.) as invasive Grade III ductal adenocarcinomas. After the exclusion of patients for whom there was no follow-up data, non-breast cancer associated deaths and unreadable IHC expression, 73 patients were available for further analysis. None of the patients had received preoperative radiochemotherapy. The characteristics of the patients and tumours is shown in Table 1, with the following definitions of breast cancer stage III:

\section{Stage IIIA}

- no tumor is found in the breast. Cancer is found in axillary lymph nodes that are attached to each other or to other structures, or cancer may be found in lymph nodes near the breastbone; or

- the tumor is 2 centimeters or smaller. Cancer has spread to axillary lymph nodes that are attached to each other or to other structures, or cancer may have spread to lymph nodes near the breastbone; or

- the tumor is larger than 2 centimeters but not larger than 5 centimeters. Cancer has spread to axillary lymph 
nodes that are attached to each other or to other structures, or cancer may have spread to lymph nodes near the breastbone; or

- the tumor is larger than 5 centimeters. Cancer has spread to axillary lymph nodes that may be attached to each other or to other structures, or cancer may have spread to lymph nodes near the breastbone.

\section{Stage IIIB}

In stage IIIB, the tumor may be any size and cancer:

- has spread to the chest wall and/or the skin of the breast; and

- may have spread to axillary lymph nodes that may be attached to each other or to other structures, or cancer may have spread to lymph nodes near the breastbone.

- Cancer that has spread to the skin of the breast is inflammatory breast cancer. See the section on Inflammatory Breast Cancer for more information.

\section{Stage IIIC}

In stage IIIC, there may be no sign of cancer in the breast or the tumor may be any size and may have spread to the chest wall and/or the skin of the breast. Also, cancer:

- has spread to lymph nodes above or below the collarbone; and

- may have spread to axillary lymph nodes or to lymph nodes near the breastbone.

- Cancer that has spread to the skin of the breast is inflammatory breast cancer. See the section on Inflammatory Breast Cancer for more information.

Stage IIIC breast cancer is divided into operable and inoperable stage IIIC.

In operable stage IIIC, the cancer:

- is found in ten or more axillary lymph nodes; or

- is found in lymph nodes below the collarbone; or

- is found in axillary lymph nodes and in lymph nodes near the breastbone.

- In inoperable stage IIIC breast cancer, the cancer has spread to the lymph nodes above the collarbone.

\section{Tissue microarray blocks and immunohistochemical staining}

Tumour-rich tissue from each biopsy was distinguished from surrounding normal tissue in $\mathrm{H} \& \mathrm{E}$-stained sections by a qualified pathologist (G.D.F). Tissue microarrays were constructed in duplicate from tumour-rich tissue cores $(1 \mathrm{~mm} \times 0.6 \mathrm{~mm})$ using an automated tissue microarray (TMA) instrument (ATA-27; Beecher Instruments). $4 \mu \mathrm{M}$ sections of the TMA blocks were used for immunohistochemical (IHC) analysis. Sections were transferred on to glass slides, deparaffinised and immunostained using anti-ER (SP1), anti-PR, (SP2), anti-
HER2/neu (4B5) (Ventana Medical Systems, pre-diluted) or anti-AR (Biocare Medical, 1:50 dilution) antibodies, and counterstained with 3,3'-Diaminobenzidine (DAB) and hematoxylin. Staining was performed with the BenchMark automated slide stainer (Ventana) using the iVIEW DAB detection kit with additional Avidin and Biotin Blockers according to the manufacturer's instructions. Analysis of stained sections was performed by a qualified pathologist (G.D.F) and the presence of tumour tissue confirmed by examining the counterstain. Expression was scored as positive when visible staining $\geq 1 \%$ was observed in the nucleus for ER, PR and AR. For HER2 IHC was semiquantitatively evaluated with a score of $3+$ regarded as positive, $2+$ as equivocal, and $1+$ or 0 as negative. In instances of an equivocal evaluation, silver-enhanced in situ hybridisation was performed as previously described [32].

\section{DNA methylation analysis}

Genomic DNA was isolated from cell lines using the NucleoSpin Tissue kit (MachereyNagel) according to the manufacturer's instructions. For each human tumour sample, four FFPE tumour-rich tissue cores $(1 \mathrm{~mm} \times$ $0.6 \mathrm{~mm}$ ) were crushed and digested with proteinase $\mathrm{K}$ at $55^{\circ} \mathrm{C}$ for 2 days and treated with $20 \mathrm{mg}$ RNase A for 1 hr at $37^{\circ} \mathrm{C}$. DNA was isolated using the PureGene kit (Qiagen) and subjected to bisulfite modification using the MethylEasy Xceed kit (Human Genetic Signatures) according to the manufacturer's instructions.

PCR amplification and methylation sensitive high resolution melt analysis (MS-HRM) was performed in duplicate on the RotorGene ${ }^{\mathrm{TM}} \mathrm{Q}$ (Qiagen). Primers were designed according to the principles outlined in [33] to control for PCR bias and are shown in Additional file 1: Table S1. PCR was performed using $2 \mathrm{ng}$ of bisulphite modified template and standard PCR conditions, followed by one cycle of $1 \mathrm{~min} 30 \mathrm{sec}$ at $72^{\circ} \mathrm{C}$ and an MSHRM step from $70^{\circ} \mathrm{C}$ to $90^{\circ} \mathrm{C}$ rising by $0.1^{\circ} \mathrm{C} / \mathrm{sec}$. Bisulfite treated CpGenome ${ }^{\mathrm{TM}}$ Universal Methylated DNA (Chemicon, Millipore) and DNA from T47D were used as positive/methylated and negative/unmethylated controls, respectively. The methylation status of these controls was confirmed by direct sequencing of MS-HRM products, purified using the QiaQuick Gel Extraction Kit (Qiagen), performed by the Australian Genome Research Facility (AGRF, Brisbane, Australia). To create a range of methylated standards, these controls were mixed in 25, 50 and $75 \%$ methylated to unmethylated template ratios and were included in the analysis of each region.

\section{Sequenom MassARRAY DNA Methylation Analysis}

Sequenom MassARRAY methylation analysis was performed as described previously [26]. The forward primer 
has a 10-mer tag (5-aggaagagag-3) and the reverse primer has a T7-promoter tag (5-cagtaatacgactcactatagggagaaggct-3) (Additional file 1: Table S1). Bisulfite treated CpGenome ${ }^{\mathrm{TM}}$ Universal Methylated DNA (Chemicon, Millipore) and Whole genome amplified DNA prepared as per instructions with the GenomePlex ${ }^{\circledR}$ Complete Whole Genome Amplification kit (Sigma) were used as positive/methylated and negative/unmethylated controls, respectively. Triplicate PCR reactions were pooled and Shrimp Alkaline Phosphatase (Sequenom, San Diego) treatment performed followed by transcription and $\mathrm{RNa}$ seA Cleavage for the T-cleavage reaction. Purified samples were nanodispensed onto silicon chips preloaded with matrix (SpectroCHIPs; Sequenom, San Diego). Mass spectra were collected using a MassARRAY mass spectrometer (Bruker-Sequenom) and results analysed by the EpiTYPER software V 1.0. Methylation readings with overlapping signals and silent peaks were eliminated from the calculation.

\section{Quantitation of AR mRNA}

To quantitate $A R$ mRNA from cell lines, total RNA was extracted using Trizol (Invitrogen). cDNA was synthesised using $500 \mathrm{ng}$ of RNA and Superscript First Strand Synthesis System III (Invitrogen), according to the manufacturer's instructions. $\beta$-actin was used to normalise mRNA concentration and primers are shown in Additional file 1: Table S1. Real-time PCR was performed in duplicate using the RotorGene ${ }^{\mathrm{TM}} \mathrm{Q}$ (Qiagen) using 50 cycles of standard PCR conditions.

\section{Somatic mutation analysis}

In breast cancer cell lines, a region spanning -659 to +280 with respect to the start of transcription $(+1)$ were examined for somatic mutations. In FFPE tumours, the fragmented nature and limited availability of DNA meant that analysis was constrained to a smaller region $(-165$ to +133$)$ and was examined in the 32 tumours for which IHC indicated negative AR expression. Primer sequences are shown in Additional file 1: Table S1. PCR was performed using KAPAHiFi DNA polymerase (KAPA Biosystems, Geneworks, Australia) and $50 \mathrm{ng}$ of template using 30 amplification cycles as per the manufacturer's instructions. PCR reactions were purified using the QiaQuick Gel Extraction Kit (Qiagen) and sequencing performed by AGRF (Brisbane, Australia).

\section{Transcription factor binding site analysis}

Bioinformatic analysis initially involved an analysis of UCSC Genome Browser (http://genome.ucsc.edu). Transcription factor binding sites were predicted by MOODS (MOtif Occurrence Detection Suite) [34]. MOODS uses the standard scoring model (log-odds against the background distribution) of PWMs. Scoring thresholds were specified by $P$-value less than or equal to 0.01 . We tested T105A 5'UTR variant and WT sequences for overlap with the TFBS models in the JASPAR database [35]. TFBS logos were downloaded from the JASPAR database web server: http://jaspar.cgb.ki.se/.

\section{$A R$ 5'UTR reporter assays}

The $A R$ 5'UTR T105A mutation was introduced into the wild-type $A R$ 5'UTR sequence (1116 bp, GenBank Accession No. NG009014.1) by site-directed mutagenesis using a two-step PCR procedure using the primers listed in Additional file 1: Table S1 and standard PCR conditions. The AR 5'UTR wild-type and T105A mutant alone and together with the $A R$ promoter were cloned into the HindIII/NcoI sites of pGL3-promoter and the KpnI/NcoI sites of pGL3-basic (Promega), respectively. Constructs were confirmed by sequencing performed by AGRF (Brisbane, Australia).

MCF7 and T47D cells were transiently transfected with $800 \mathrm{ng}$ pGL3+/- either the SV40 or the $A R$ promoter, together with either the wild-type or mutant $A R$ 5'UTR sequence and $100 \mathrm{ng}$ Renilla reporter in a 24well plate, using Lipofectamine 2000 (Invitrogen). Fortyeight hours after the initial transfections, relative luciferase activities were determined using the Dual-Glo luciferase assay kit (Promega) and a DTX880 Multimode Detector (Beckman Coulter) according to the manufacturer's instructions. Statistical analysis was performed using unpaired, two-tailed t tests, with $\mathrm{p}$ values $<0.05$ considered significant.

\section{miRNA analysis}

Two algorithms, miRanda and TargetScan 5.1, were used to predict target sites for miRNA in the AR 3'UTR $[36,37]$. A total of 55 miRNA were predicted to target AR, 12 of which have conserved seed sequences. Only miR-124 was predicted by both algorithms. To quantitate the expression of miR-124, total RNA from transfected MCF7 and T47D cells was extracted using Trizol (Invitrogen) and the expression of miR-124 determined relative to RNU6B using the miScript PCR System according to the manufacturer's instructions (Qiagen).

\section{$A R$ 3'UTR reporter assays}

The AR 3'UTR (436 bp; GenBank Accession No. NG009014.1) was ligated downstream of the luciferase coding sequence in the pSG5 vector (Stratagene). The mir-124-1 stem-loop (84 bp; miRBase Accession No. MI0000443) \pm 200 bp of flanking sequences was cloned into the KpnI/XbaI sites of the pcDNA 3.1(+) expression vector (Invitrogen). MCF7 and T47D cells were transiently transfected with 400 ng pcDNA 3.1(+) constructs, 200 ng pSG5-luciferase constructs and 10 ng of Renilla reporter in a 24-well plate, using FuGene 6 (Roche). 
Forty-eight hours after the initial transfections, relative luciferase activities were determined using the Dual-Glo luciferase assay kit (Promega) and a DTX880 Multimode Detector (Beckman Coulter) according to the manufacturer's instructions. Statistical analysis was performed using unpaired, two-tailed $\mathrm{t}$ tests, with $\mathrm{p}$ values $<0.05$ considered significant.

\section{Statistical analysis}

Survival time was calculated from the date of tumour removal to the date of last follow-up or death attributable to breast cancer. Overall survival probabilities were estimated non-parametrically with the use of the Kaplan-Meier product limit method (GraphPad Prism version 5.0a) and statistical significance accepted as $\mathrm{P} \leq$ 0.05 .

\section{Additional material}

Additional file 1: Table S1. Oligonucleotide primers.

\section{Acknowledgements}

This project was funded by grants from the National Breast Cancer Foundation (NBCF), Cancer Australia and National Health and Medical Research Council (NHMRC). SE was supported by an NHMRC CJ Martin Fellowship.

\section{Author details \\ 'School of Chemistry and Molecular Biosciences, The University of Queensland, St. Lucia 4072 Queensland, Australia. ²Epigenetics Group, Garvan Institute of Medical Research, Sydney 2010, NSW, Australia. ${ }^{3}$ Department of Pathology, Princess Alexandra Hospital, Queensland Woolloongabba, 4102 Queensland, Australia.}

\section{Authors' contributions}

Kate M Peters ${ }^{1}$ : Contributed to AR IHC analysis, developed AR MS-HRM assay, determined expression levels in Breast cancer cell-lines. Drafted manuscript. Stacey L Edwards' ${ }^{1}$ : Performed AR 5'UTR reporter assays. Shalima S Nair ${ }^{2}$. Performed AR Sequenom methylation assays on clinical samples and celllines. Juliet D French ${ }^{1}$ : Peter J Bailey ${ }^{1}$ : performed bioinformatics analysis of AR 5'UTR and promoter region. Kathryn Salkield': Contributed to AR IHC analysis. Sandra Stein ${ }^{3}$ : Managed breast tumour biobank, created tumour tissue array, optimized AR antibody $I H C$, performed AR, ER, PR and HER2 IHC. Sarah Wagner ${ }^{3}$ : Contributed to creation of tumour tissue array and optimization of AR antibody IHC. Glenn D Francis ${ }^{3}$ : Pathologist responsible to tumour bank, pathological review of tumour samples and analysis of $E R$, PR, HER2 and AR IHC results. Susan J Clark²: Contributed to project design, attracting research funding, interpreting results and review of manuscript. Melissa A Brown ${ }^{1}$ Project leader. Contributed to project design, attracting research funding, interpreting results and reviewing, editing, submission and post-review revision of manuscript. All authors read and approved the final manuscript.

\section{Competing interests}

The authors declare that they have no competing interests.

Received: 7 December 2011 Accepted: 2 April 2012

Published: 2 April 2012

\section{References}

1. Sorlie T, Perou CM, Tibshirani R, Aas T, Geisler S, Johnsen H, Hastie T, Eisen MB, van de Rijn M, Jeffrey SS, et al: Gene expression patterns of breast carcinomas distinguish tumor subclasses with clinical implications. Proc Natl Acad Sci USA 2001, 98(19):10869-10874.

2. Rakha EA, Reis-Filho JS, Ellis IO: Combinatorial biomarker expression in breast cancer. Breast Cancer Res Treat 2010, 120(2):293-308.

3. Yeh S, Hu YC, Wang PH, Xie C, Xu Q, Tsai MY, Dong Z, Wang RS, Lee TH, Chang C: Abnormal mammary gland development and growth retardation in female mice and MCF7 breast cancer cells lacking androgen receptor. J Exp Med 2003, 198(12):1899-1908.

4. Liao DJ, Dickson RB: Roles of androgens in the development, growth, and carcinogenesis of the mammary gland. J Steroid Biochem Mol Biol 2002, 80(2):175-189.

5. Li S, Han B, Liu G, Ouellet J, Labrie F, Pelletier G: Immunocytochemical localization of sex steroid hormone receptors in normal human mammary gland. J Histochem Cytochem 2010, 58(6):509-515.

6. Doane AS, Danso M, Lal P, Donaton M, Zhang L, Hudis C, Gerald WL: An estrogen receptor-negative breast cancer subset characterized by a hormonally regulated transcriptional program and response to androgen. Oncogene 2006, 25(28):3994-4008.

7. Birrell SN, Bentel JM, Hickey TE, Ricciardelli C, Weger MA, Horsfall DJ, Tilley WD: Androgens induce divergent proliferative responses in human breast cancer cell lines. J Steroid Biochem Mol Biol 1995, 52(5):459-467.

8. Ni M, Chen Y, Lim E, Wimberly H, Bailey ST, Imai Y, Rimm DL, Liu XS, Brown M: Targeting androgen receptor in estrogen receptor-negative breast cancer. Cancer Cell 2011, 20:119-131.

9. Agoff SN, Swanson PE, Linden H, Hawes SE, Lawton TJ: Androgen receptor expression in estrogen receptor-negative breast cancer. Immunohistochemical, clinical, and prognostic associations. Am J Clin Pathol 2003, 120(5):725-731.

10. Gonzalez-Angulo AM, Stemke-Hale K, Palla SL, Carey M, Agarwal R, MericBerstam F, Traina TA, Hudis C, Hortobagyi GN, Gerald WL, et al: Androgen receptor levels and association with PIK3CA mutations and prognosis in breast cancer. Clin Cancer Res 2009, 15(7):2472-2478.

11. Faber PW, van Rooij HC, van der Korput HA, Baarends WM, Brinkmann AO, Grootegoed JA, Trapman J: Characterization of the human androgen receptor transcription unit. J Biol Chem 1991, 266(17):10743-10749.

12. Faber PW, van Rooij HC, Schipper HJ, Brinkmann AO, Trapman J: Two different, overlapping pathways of transcription initiation are active on the TATA-less human androgen receptor promoter. The role of Sp1. J Biol Chem 1993, 268(13):9296-9301.

13. Chen S, Supakar PC, Vellanoweth RL, Song CS, Chatterjee B, Roy AK: Functional role of a conformationally flexible homopurine/ homopyrimidine domain of the androgen receptor gene promoter interacting with Sp1 and a pyrimidine single strand DNA-binding protein. Mol Endocrinol 1997, 11(1):3-15

14. Takane KK, McPhaul MJ: Functional analysis of the human androgen receptor promoter. Mol Cell Endocrinol 1996, 119(1):83-93.

15. Mizokami A, Yeh SY, Chang C: Identification of 3',5'-cyclic adenosine monophosphate response element and other cis-acting elements in the human androgen receptor gene promoter. Mol Endocrinol 1994, 8(1):77-88.

16. Kinoshita H, Shi Y, Sandefur C, Meisner LF, Chang C, Choon A, Reznikoff CR, Bova GS, Friedl A, Jarrard DF: Methylation of the androgen receptor minimal promoter silences transcription in human prostate cancer. Cancer Res 2000, 60(13):3623-3630.

17. Waltering KK, Wallen MJ, Tammela TL, Vessella RL, Visakorpi T: Mutation screening of the androgen receptor promoter and untranslated regions in prostate cancer. Prostate 2006, 66(15):1585-1591.

18. Crocitto LE, Henderson BE, Coetzee GA: Identification of two germline point mutations in the $5^{\prime} \mathrm{UTR}$ of the androgen receptor gene in men with prostate cancer. J Urol 1997, 158(4):1599-1601.

19. Cox DG, Blanche H, Pearce CL, Calle EE, Colditz GA, Pike MC, Albanes D, Allen NE, Amiano P, Berglund $G$, et al: A comprehensive analysis of the androgen receptor gene and risk of breast cancer: results from the National Cancer Institute Breast and Prostate Cancer Cohort Consortium (BPC3). Breast Cancer Res 2006, 8(5):R54.

20. Kyndi M, Sorensen FB, Knudsen H, Overgaard M, Nielsen HM, Andersen J, Overgaard J: Tissue microarrays compared with whole sections and biochemical analyses. A subgroup analysis of DBCG 82 b\&c. Acta Oncol 2008, 47(4):591-599. 
21. Karlsson C, Bodin L, Piehl-Aulin K, Karlsson MG: Tissue Microarray Validation: A Methodologic Study with Special Reference to Lung Cancer. Cancer Epidemiology Biomarkers \& Prevention 2009, 18:2014-2021.

22. Castellano I, Allia E, Accortanzo V, Vandone AM, Chiusa L, Arisio R, Durando A, Donadio M, Bussolati G, Coates AS, et al: Androgen receptor expression is a significant prognostic factor in estrogen receptor positive breast cancers. Breast Cancer Res Treat 2010.

23. Bryan RM, Mercer RJ, Bennett RC, Rennie GC, Lie TH, Morgan FJ: Androgen receptors in breast cancer. Cancer 1984, 54(11):2436-2440.

24. Park S, Koo JS, Kim MS, Park HS, Lee JS, Kim SI, Park BW, Lee KS: Androgen receptor expression is significantly associated with better outcomes in estrogen receptor-positive breast cancers. Ann Oncol 2011.

25. Jarrard DF, Kinoshita H, Shi Y, Sandefur C, Hoff D, Meisner LF, Chang C, Herman JG, Isaacs WB, Nassif N: Methylation of the androgen receptor promoter CpG island is associated with loss of androgen receptor expression in prostate cancer cells. Cancer Res 1998, 58(23):5310-5314.

26. Coolen MW, Statham AL, Gardiner-Garden M, Clark SJ: Genomic profiling of CpG methylation and allelic specificity using quantitative highthroughput mass spectrometry: critical evaluation and improvements. Nucleic Acids Res 2007, 35(18):e119.

27. Yeap BB, Wilce JA, Leedman PJ: The androgen receptor mRNA. Bioessays 2004, 26(6):672-682.

28. Hurst DR, Edmonds MD, Welch DR: Metastamir: the field of metastasisregulatory microRNA is spreading. Cancer Research 2009, 69(19):7495-7498.

29. Valastyan S, Benaich N, Chang A, Reinhardt F, Weinberg RA: Concomitant suppression of three target genes can explain the impact of a microRNA on metastasis. Genes Dev 2009, 23(22):2592-2597.

30. Sikand K, Slaibi JE, Singh R, Slane SD, Shukla GC: miR 488* inhibits androgen receptor expression in prostate carcinoma cells. Int J Cancer 2010.

31. Neve RM, Chin K, Fridlyand J, Yeh J, Baehner FL, Fevr T, Clark L, Bayani N, Coppe JP, Tong F, et al: A collection of breast cancer cell lines for the study of functionally distinct cancer subtypes. Cancer Cell 2006, 10(6):515-527.

32. Francis GD, Jones MA, Beadle GF, Stein SR: Bright-field in situ hybridization for HER2 gene amplification in breast cancer using tissue microarrays: correlation between chromogenic (CISH) and automated silver-enhanced (SISH) methods with patient outcome. Diagn Mol Pathol 2009, 18:88-95.

33. Wojdacz TK, Hansen $L L$, Dobrovic A: A new approach to primer design for the control of PCR bias in methylation studies. BMC Res Notes 2008, 1:54.

34. Korhonen J, Martinmäki P, Pizzi CPR, Ukkonen E: MOODS: fast search for position weight matrix matches in DNA sequences. Bioinformatics 2009, 25:3181-3182.

35. Bryne JC, Valen E, Tang MH, Marstrand T, Winther O, da Piedade I, Krogh A, Lenhard B, Sandelin A: JASPAR, the open access database of transcription factor-binding profiles: new content and tools in the 2008 update. Nucleic Acids Res 2008, 36:102-106.

36. Miranda KC, Huynh T, Tay Y, Ang YS, Tam WL, Thomson AM, Lim B, Rigoutsos I: A pattern-based method for the identification of MicroRNA binding sites and their corresponding heteroduplexes. Cell 2006, 126(6):1203-1217.

37. Lewis BP, Burge CB, Bartel DP: Conserved seed pairing, often flanked by adenosines, indicates that thousands of human genes are microRNA targets. Cell 2005, 120(1):15-20.

\section{Pre-publication history}

The pre-publication history for this paper can be accessed here: http://www.biomedcentral.com/1471-2407/12/132/prepub

\section{doi:10.1186/1471-2407-12-132}

Cite this article as: Peters et al:: Androgen receptor expression predicts breast cancer survival: the role of genetic and epigenetic events. BMC Cancer 2012 12:132.

\section{Submit your next manuscript to BioMed Central and take full advantage of:}

- Convenient online submission

- Thorough peer review

- No space constraints or color figure charges

- Immediate publication on acceptance

- Inclusion in PubMed, CAS, Scopus and Google Scholar

- Research which is freely available for redistribution

Submit your manuscript at www.biomedcentral.com/submit
Biomed Central 